\title{
Sharing Incentivization Through Flexible Spectrum Licensing
}

\author{
Arturo Basaure \\ Aalto University. Espoo, Finland \\ arturo.basaure@aalto.fi
}

\begin{abstract}
Recent developments in radio access technologies are radically changing the management of the spectrum, progressing from exclusive licensing with static conditions towards more flexible licensing schemes which allow dynamic spectrum assignment. In this context, spectrum transactions between participating actors should generate mutual benefits. However, the fact that spectrum sharing increases the interference between users requires a clear definition of this interference and any associated mutual benefits. This paper proposes a contract structure between a primary and a secondary operator, which considers different service requirements in the context of spectrum transactions. The simulated contracts and transactions suggest that (i) the optimal level of interference is usually positive, and (ii) given a spectrum size, an increase in the demand results in additional gains in a scheme which allows voluntary transactions with respect to a scheme, which do not allow or minimize the interference.
\end{abstract}

Keywords: Spectrum sharing; Flexible spectrum licensing; Measuring and pricing interference; Signal-to-interference and noise ratio (SINR); end user utility function; agent-based modeling

\section{INTRODUCTION}

Spectrum management has been raised as one of the main challenges of nowadays mobile communications, especially in the context of constant evolution. Latest development in Radio Access Technologies (RAT) enables to assign spectrum licenses with more flexible conditions. However, spectrum holders (i.e. mobile operators) usually have not been enthusiastic in supporting spectrum sharing ${ }^{1}$. Thus, understanding the incentives of the spectrum holder will play an important role when implementing new spectrum regimes.

During last years, governments performing spectrum auctions have achieved very different outcomes. Given this, the possibility of sharing spectrum more dynamically has been suggested as a means to achieve more accurate valuations. Since agents valuate spectrum differently, spectrum transactions should generate gains. A spectrum market has the role of matching supply with demand to reach an optimal spectrum allocation. Nevertheless, a secondary usage of the spectrum generates interference to the primary users, and therefore transactions need to consider this negative externality. Thus, the ability of measuring interference will determine how prices can internalize it.

\footnotetext{
${ }^{1}$ In this paper, spectrum sharing refers to the technology enabling different type of users to co-exist in a frequency band. Spectrum trading refers to the monetary value paid in exchange for accessing this frequency band.
}

\author{
Oliver Holland \\ King's College London. London, UK \\ oliver.holland@kcl.ac.uk
}

The literature presents two opposing market types namely, reservation and spot markets. While a reservation market is static and performed through long term contracts, a spot market is much more dynamic and user driven, usually based on real-time retail pricing. From this perspective, spectrum trading may evolve towards a spot market along with the development of the technology. Analogically, in energy markets the fluctuations of different sources of energy, especially in the case of renewable energy sources, limits the benefits of market based mechanisms [1]. Spot markets seem to fit better for a low-risk scenario with low fluctuations. These markets have been widely investigated in the literature, for example by [2], [3] and [4]. A study on the impact of reservation markets can be found in [5].

Several new spectrum regimes have been proposed to incentivize sharing. Licensed Shared Access (LSA) is a licensing regime, which permits the spectrum holder leasing its spectrum to additional users with a guaranteed quality of service, through a legal contract providing certainty and financial compensation [6]. Light licensing [7] provides a collective access of the spectrum, assuring a certain quality of service by requesting a registration mechanism or other means of coordination (also referred as to private commons). While LSA has come from the industry and initially focused on getting additional spectrum to mobile operators, light licensing has been proposed by the European Commission. Finally and notably, pluralistic licensing [8] proposes a flexible licensing mechanism, which allows the spectrum holder to adapt its interference tolerance in exchange for a monetary compensation based on well-known and predictable interference results. This regime considers explicitly the interference in the license price. In fact, pluralistic licensing requires the primary operator to agree the interference parameters and rules at the point of obtaining the license. Such a license incentivizes the operator to accept secondary users through a reduced license fee or additional revenue mechanism, whereby the secondary spectrum access will be based in cognitive radio system (CRS) mechanisms to avoid causing interference to the primary or to otherwise keep interference within known parameters.

The current literature lacks understanding in determining the spectrum value from a user perspective, which includes the interference measured by the state-of-the-art technology and the user valuation. An attempt to valuate according to the experienced quality of service (QoS) can be found in [9], which analyses theoretical quality-price contracts between primary and multiple secondary users. Another theoretical 
approach [10] explores different operator pricing strategies for spectrum trading. Finally, [11] studies the conditions of profitability for trading spectrum through private commons regime. This paper explores the concept of pluralistic licensing as a means to measure and valuate the interference when trading spectrum. Previous related work has been theoretical. With the purpose of understanding the real implications of such a regime, this study carries out realistic simulations together with a sensitivity analysis that provide useful guidelines on pricing decisions.

In concrete, this paper aims to define a contract structure for a flexible spectrum licensing scheme in which a spectrum database authorizes the secondary access, taking into consideration the interference and the user valuation.

\section{A MODEL FOR VALUATING INTERFERENCE}

This section develops a model which simulates the interference experienced by primary and secondary users, to understand the incentives and conditions for spectrum trading.

To date, interference measurement has been extremely challenging, although it is improving along with the development of Cognitive Radio Systems (CRS) and Software Defined Radio (SDR) technologies. Although several sensing techniques have been developed [12], its current state-of-theart presents important challenges, such as the sensing time and delay, cooperation and energy efficiency, tracking mobile users and channel impairment [13]. Nevertheless, secondary spectrum access can be presently based on an authoritative register (here referred as to spectrum database), from which users request permission for accessing the spectrum. This database has predefined rules based on not real-time interference measurement. For instance, an example of such rules and requirements for a spectrum database can be found in [14] and [15]. Such a spectrum database holds the statistical information on interference, which will improve in accuracy with SDR technologies [16].

This study models a spectrum sharing scheme based on primary-secondary coexistence, in which the primary and secondary services have different requirements. The analysis herein is intended to be a reflection of [8] as a method of facilitating and compensating in for flexibility in spectrum use permitted by the primary spectrum owner, although may be more generally extrapolated to other schemes. Moreover, the technical characteristics and geographical location of the primary user are known by the spectrum database. The secondary device will request permission from the spectrum database, which allows the secondary user to transmit if his/her location is within a predefined area (here referred as to coverage area) and if he/she moves within a predefined radius (here referred as to leasing distance). Thus, while primary users are always allowed to transmit within their coverage area, secondary users can transmit only if their access request is accepted and with limited mobility. A secondary user can be additionally equipped with more advanced sensing capabilities or any other mean to achieve primary awareness to guarantee coexistence without producing harmful interference. This work assumes that the secondary awareness will be based on the authorization given by the spectrum database, without involving real-time awareness, which will further improve the performance of the system.

The main challenge for assuring coexistence is the interference measurements. Once this is solved correctly, the monetary compensation could be transferred by a centralized entity or directly from the secondary users.

This section develops a simulation model to understand the interference behavior for primary and secondary users coexisting in the $700 \mathrm{MHz}$ band. This model represents an urban area, in which the primary system consists of a 7-cell cluster of LTE type cellular network, modeled as a 5-cell at the edge of coverage. The secondary system is modelled as a set of peer-to-peer (P2P) terminals, which do not require base stations and have a different coverage area than the primaries (Figure 1, left).

The simulation is performed by employing agent based modeling $(\mathrm{ABM})$. ABM is especially suitable for modeling the behavior of agents (such as end users) to observe the collective effect of their interaction [17]. This study analyzes the overall impact of primary and secondary systems to every single user.

The model focuses on the behavior of the users located near the base station of the center, because they are surrounded by the rest of the base stations and thus represent better the reality. Primary base stations are indicated in the diagram with larger arrows and end users with smaller arrows. The secondary users can transmit in the same frequency band in a limited area located outside the 5-cell cluster, as indicated in the Figure 1(left).

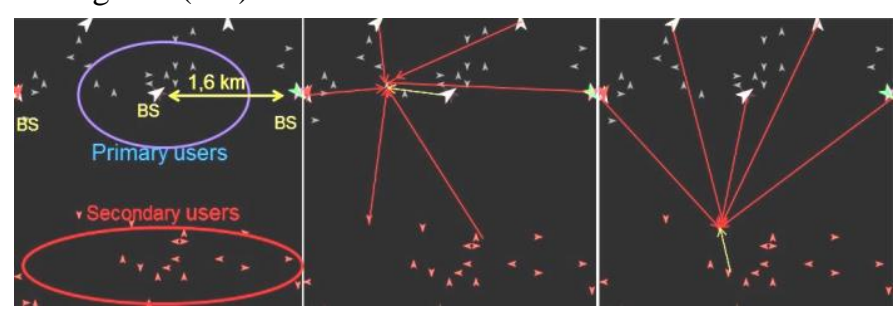

Figure 1: (Left) simulation setup diagram. (Middle and right) graphical representation of the primary (middle) and secondary (right) transmissions (signal in yellow and interference in red).

Table 1: Assumptions of the model and simulation setup

\begin{tabular}{|c|c|}
\hline \multicolumn{2}{|l|}{ Assumptions } \\
\hline Parameter & Value \\
\hline Primary BS spacing & $1-1.6 \mathrm{~km}$ \\
\hline Secondary leasing distance & 100,200 and $300 \mathrm{~m}$ \\
\hline Separation between coverage areas & $700 \mathrm{~m}$ \\
\hline BS transmission centre frequency & $700 \mathrm{MHz}$ \\
\hline Primary BS transmission power & from 40 to $53 \mathrm{dBm}$ \\
\hline Primary terminals transmission power & $21 \mathrm{dBm}$ \\
\hline Secondary terminals transmission power & $20 \mathrm{dBm}$ \\
\hline Path loss model from Base Station to terminal & Cost 231 Hata model [18] \\
\hline Path loss model from terminal to terminal & ITU-R P.1411 [19] \\
\hline Noise power & $\begin{array}{l}-105 \mathrm{dBm} \text { or } 0.032 \mathrm{pW} \\
\text { (in } 8 \mathrm{MHz} \text { channel) }\end{array}$ \\
\hline Shadowing standard deviation & 6 \\
\hline BS effective height, primary case & $30 \mathrm{~m}$ \\
\hline $\begin{array}{l}\text { Primary terminal and secondary terminal } \\
\text { effective height }\end{array}$ & $1.5 \mathrm{~m}$ \\
\hline Mobile node effective height & $1.5 \mathrm{~m}$ \\
\hline
\end{tabular}


Table 1 describes the simulation assumptions for the technical and location-related parameters. The database holds information on the interference the primary is able to receive, which depends on the transmission power of the primary base station. The permission is granted to the secondary if the additional interference is within predefined limits. In practice, interference figures are described as distributions, which might vary over time.

At each simulation cycle, all users move randomly (following a random walk algorithm). After the primary system attends the user demands, secondary users request access from the spectrum database. Permission to transmit and receive is given to the secondary with a mobility restriction (leasing distance), after which the secondary user should request again for accessing the spectrum. All users require a minimum amount of signal-to-interference-and-noise ratio (SINR) to be considered as active users. Otherwise, they are out of range.

In specific, the model analyzes the interference behavior of the downlink, which is being utilized by the primary and the secondary users (based on frequency division duplex, FDD). Figure 1 (middle, right) describes the signal and the interference with different colors (yellow and red, respectively). To assess the overall externality caused by interference, the model quantifies the received signal and interference received at each end user terminal by employing a suitable path loss formula. The COST 231 Hata propagation model for urban areas [18] is adopted for determining the signal and interference transmitted from primary base station. The propagation model of ITU Recommendation P.1411 [19] is adopted for determining the terminal to terminal communication, using a $99 \%$ of error rate probability. This model supposes a high level coordination between secondary end user terminals, in such a way that they avoid interfering to each other. In addition, the terminals have certain level of orthogonality, which is approximated by a factor of $10 \%$.

\section{SIMULATION RESULTS}

This section presents the simulation results, which will be utilized by the next section to analyze the incentives for sharing spectrum.

These simulations consist of a sensitivity analysis of the main parameters affecting the experienced interference: primary base station transmission power and secondary leasing distance, to analyze the performance of the spectrum usage in terms of total amount of users in range and the received QoS, in terms of SINR.

In concrete, simulations gradually adjust the transmission power of the primary base station from $40 \mathrm{dBm}$ to $53 \mathrm{dBm}$ (from 10 to $200 \mathrm{~W}$ ) to analyze the impact on SINR and number of active users. Additionally, they adjust the leasing distance by $+100 \mathrm{~m}$ and $+200 \mathrm{~m}$ (from the default value of $100 \mathrm{~m})$.

Simulation results are summarized in Figure 2. Figure 2 (a) depicts the cumulative distribution functions of the experienced SINR of the primary users for different primary base stations transmission power values. The total number of users does not vary with the transmission power; however, it increases by rising the leasing distance (Figure 2 (b)) at the primary expenses. The experienced SINR by the primary user suffers a small drop in its average value (Figure 2 (c)) and a larger rise in standard deviation (Figure 2 (d)), which can be understood as a higher interference uncertainty. This behavior evidences a trade-off between the secondary mobility and the a)
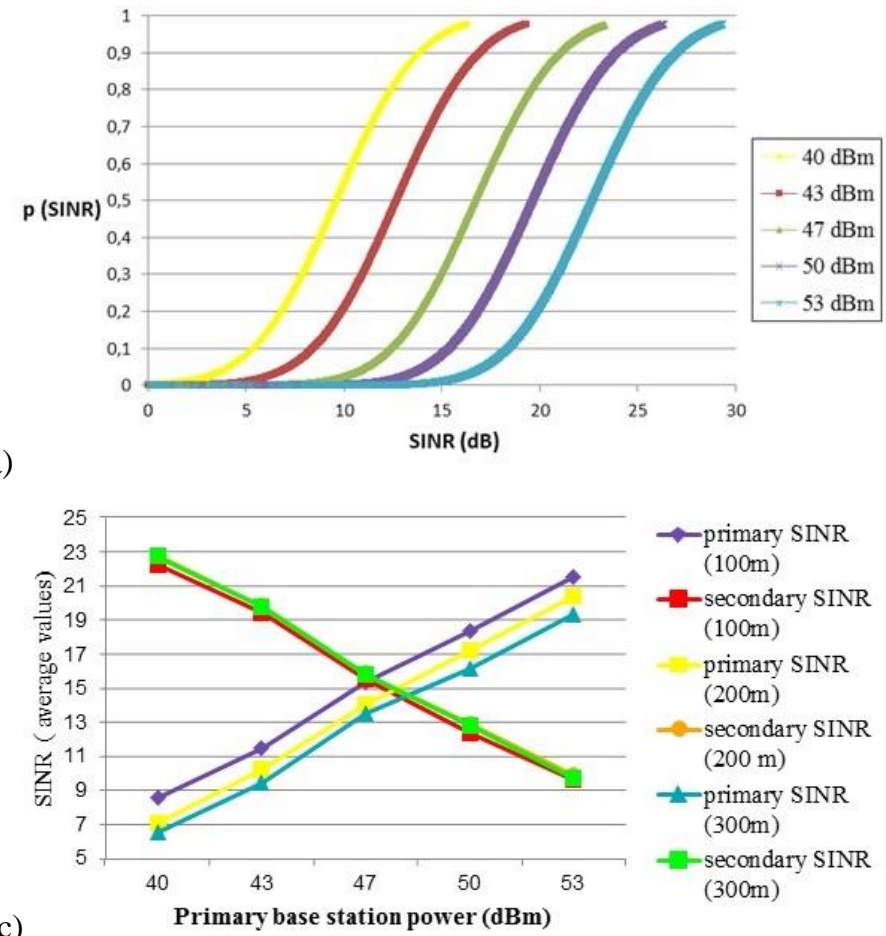

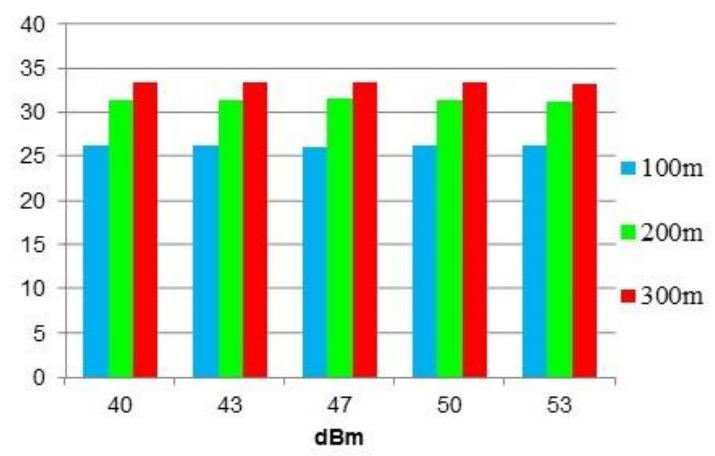

b)

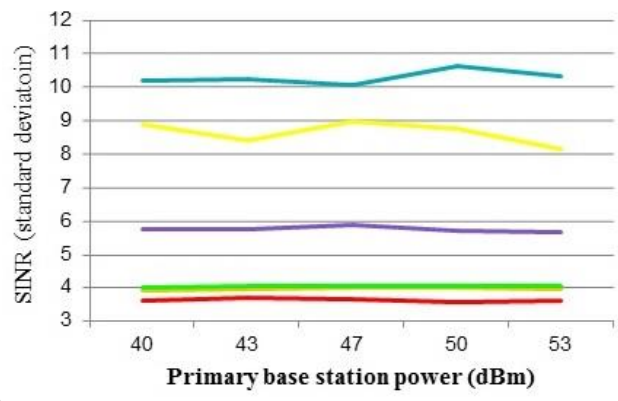

d)

Figure 2: (a) Cumulative distribution function (CDF) for the experienced SINR by primary users. (b) Total number of users against primary transmission power. (c) Average value and (d) standard deviation of SINR for primary and secondary users against transmission power. Graphs (b), (c) and (d) include a sensitivity analysis for different leasing distances $(100,200$ and 300m). 
primary QoS. However, this situation may change with the improvement of secondary sensing capabilities.

\section{INCENTIVES FOR SHARING}

This section evaluates the incentives for sharing spectrum in a flexible licensing regime, by employing the sensitivity analysis of the previous section.

Under a primary-secondary scheme, the primary system decides on the level of interference it wants to generate by adjusting its transmission power and thus deciding on the level of SINR experienced by the primary and secondary users.

From a user perspective, primary and secondary services are presumably different in nature and consequently they should require different QoS. To address this issue, this work employs a general classification of services which consider the criticality of real-time to valuate the experienced quality (SINR). The utility functions are defined as follows [20]:

$$
\begin{aligned}
& \mathrm{u}_{\text {not real-time }}=1-\mathrm{e}^{-\mathrm{k} 0 * \operatorname{SINR} /(\operatorname{SINRmax})}=1-\mathrm{e}^{-6 * \operatorname{SINR} /(30)} \\
& \mathrm{u}_{\text {real-time }}=1-\mathrm{e}^{-\mathrm{k} 1 * \operatorname{SINR}^{2} /(\mathrm{k} 2+\operatorname{SINR})}=1-\mathrm{e}^{-0.5 * \operatorname{SINR}^{2} /(100+\operatorname{SINR})} \\
& \mathrm{u}_{\text {strict real-time }}=1 \text {, when } \mathrm{SINR} \geq \mathrm{SINR}_{\text {threshold }} \\
& 0 \text {, when } \text { SINR }_{2} \mathrm{SINR}_{\text {threshold }}
\end{aligned}
$$

$\mathrm{SINR}_{\text {max }}$ is assumed to be $30 \mathrm{~dB}$, based on the simulation results (maximum achieved value at Figure 2 (a)). For the strict real-time case, the threshold value for SINR $\left(\mathrm{SINR}_{\text {threshold }}\right.$ ) is $10 \mathrm{~dB}$ (which approximately corresponds to a throughput of $35 \mathrm{Mbps}$ based on [21]). The constants $\mathrm{k}_{0}, \mathrm{k}_{1}$ and $\mathrm{k}_{2}$ define the shape of the utility function curves. Herein, these constant are set as 6, 0.5 and 100 respectively and the resulting graphical representation is shown in Figure 3.

The interference level, which attains an optimal spectrum usage, should consider the user utility functions. Thus, the

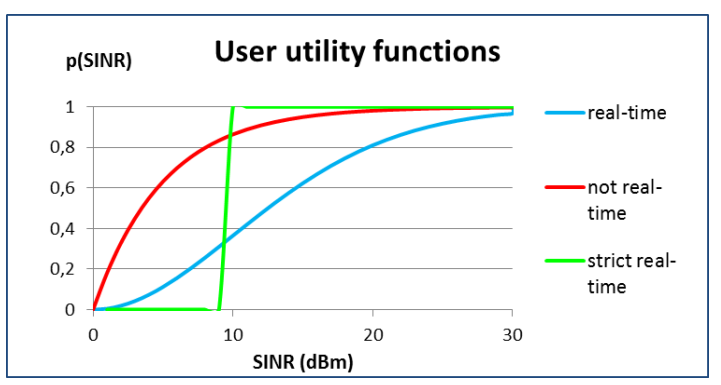

Figure 3: user utility functions for accessing spectrum for different types of usage: not real-time, real-time and strict real-time

total operator profits include herein both types of users. Moreover, if a license fee reduction is applied to the primary, it should be proportional to the secondary revenues.

The profits of the primary operator can be expressed as:

$\mathrm{P}^{\mathrm{p}}=\mathrm{R}^{\mathrm{p}}+\mathrm{R}^{\mathrm{s}}-\mathrm{C}^{\mathrm{sh}}$,

where $\mathrm{P}^{\mathrm{p}}$ : total profits; $R^{p}$ : revenue from primary users; $R^{s}$ : revenue from secondary user, and $C^{\text {sh }}$ : costs of sharing.

Additionally, the price $p$ of the service is proportional to the utility function, and then the profit equation is formulated as follows:

$\mathrm{P}=\mathrm{p}_{\max } * \Sigma_{\mathrm{i}=0}{ }^{\mathrm{N}} \mathrm{u}\left(\mathrm{SINR}_{\mathrm{i}}\right)+\mathrm{p}_{\max } * \Sigma_{\mathrm{i}=0}^{\mathrm{M}} \mathrm{u}\left(\mathrm{SINR}_{\mathrm{i}}\right)-\mathrm{C}^{\text {sh }}$

$\mathrm{P}=\mathrm{p}_{\max } * \mathrm{~N} * \mathrm{u}\left(\mathrm{SINR}_{\text {average }}\right)+\mathrm{p}_{\max } * \mathrm{M} * \mathrm{u}\left(\mathrm{SINR}_{\text {average }}\right)-\mathrm{C}^{\mathrm{sh}}$

where: N: number of primary users, $\mathrm{M}$ : number of secondary users, $\mathrm{p}_{\max }$ : price that user is willing to pay for $\mathrm{u}(\mathrm{SINR})=1$.

Note that this model assumes, for simplicity, that all primary users possess the same utility function, while this being different to the secondary utility function.

The sharing costs are approximated by the additional energy costs the primary operator incurs when adjusting its

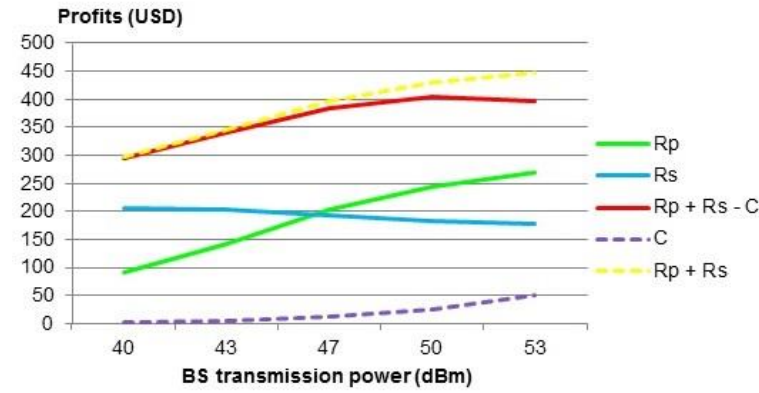

a) real-time primary / not real-time secondary

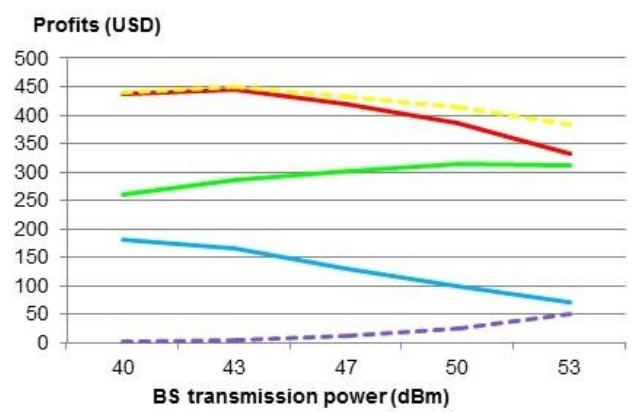

b) not real-time primary / real-time secondary

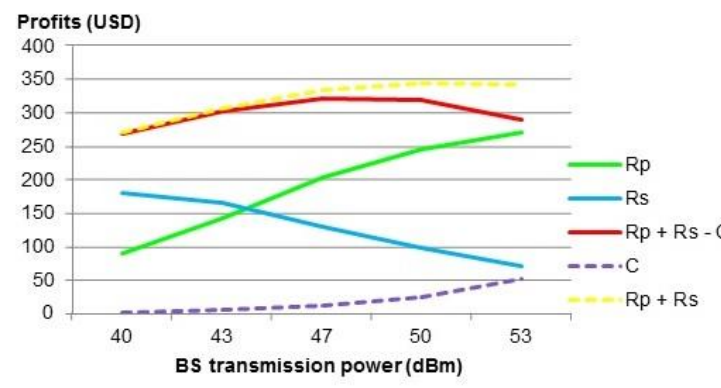

c) real-time primary and secondary

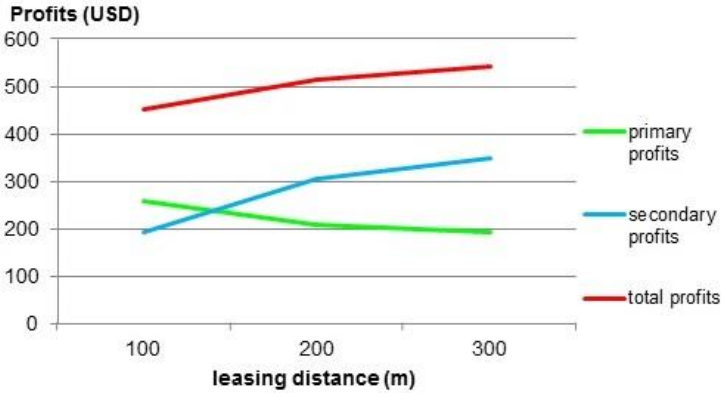

d) strict real-time primary / not real-time secondary

Figure 4: Sensitivity analysis for profit (in USD) against primary base station transmission power ((a), (b) and (c)) and leasing distance (d). 
transmission power to meet the required QoS. These energy costs are obtained by applying the following formula [22]:

$\mathrm{P}_{\text {in }}=\mathrm{N}_{\text {trx }} *\left(\mathrm{P}_{\mathrm{o}}+\Delta_{\mathrm{p}} \mathrm{P}_{\text {out }}\right) \mathrm{r}^{2}, \quad 0<\mathrm{P}_{\text {out }} \leq \mathrm{P}_{\max }$,

where $\mathrm{N}_{\text {trx }}=6, \mathrm{P}_{\mathrm{o}}=130 \mathrm{~W}, \Delta_{\mathrm{p}}=3$.

By replacing the previous utility function (1), (2) and (3), and costs (6) equations into the profit equation (5); we can derive the final expression for total spectrum holder profits. In general, the profit optimization is achieved by setting the equation's derivative to zero $(\delta \mathrm{P} / \delta(\mathrm{SINR})=0)$. Nevertheless, this paper focuses on analyzing the resulting profits for different scenarios rather than on formulating a final expression. Thus, Figure 4 illustrates the profit analysis for different utility functions. Total profits are represented by a red line; primary, secondary profits $\left(\mathrm{R}^{\mathrm{p}}, \mathrm{R}^{\mathrm{s}}\right)$ and cost of sharing $\left(\mathrm{C}^{\mathrm{sh}}\right)$ are represented by green, blue and violet lines respectively. These results indicate that the optimal transmission power varies depending on the utility function of the users. Results are calculated in USD, assuming a 20 USD monthly ARPU for a customer reaching $\mathrm{u}(\mathrm{SINR})=1$.

When primary and secondary users present the same utility function, the optimal transmission power approaches an equilibrium value, which provides every user the same SINR level $(47 \mathrm{dBm}$ in Figure 4 (c)). Nonetheless, this value may slightly change with a variation in the amount of primary and secondary users. If the primary user requires a real-time service and the secondary user a not real-time service, the optimal transmission power would be higher than $47 \mathrm{dBm}$ (50 $\mathrm{dBm}$ in Figure 4 (a)). Consequently, if the secondary user requires a real-time service and the primary user requires a not real-time service, the optimal transmission power would be lower than $47 \mathrm{dBm}$ (43 dBm in Figure 4, B). Finally, Figure 4 (d) depicts the profits resulting from the sensitivity analysis of leasing distance, assuming for this case a strict real-time requirement for primary users (being SINR $_{\text {threshold }} 10 \mathrm{~dB}$ as stated before) and a not real-time requirement for secondary users. This result evidences that profits rise along with the leasing distance, since revenues coming from secondary users exceed the losses coming from primary users.

\section{PRICING INTERFERENCE}

The previous section shows that the spectrum holder can maximize its profits by adapting its transmission power. These calculations assume that the operator knows the user spectrum valuations (i.e. both type of users are customers). However, this may not be the case, for instance, if the secondary user belongs to another operator and the spectrum holder ignores his/her utility functions.

Therefore, a secondary access pricing, which considers the caused interference as negative externality, helps revealing the secondary valuation. In the literature, congestion pricing is employed to assess different negative externalities such as pollution or traffic, e.g. to price the access of cars to highways. In congestion pricing, the price is increased in peak hours to achieve a socially optimal level of traffic.
In theory, the price due to externality should represent a marginal decrease in the individual utility due to congestion for an incremental increase in usage [23]. The average cost of each user in the system varies with the number of total users. If one additional user enters to the system (i.e. start transmitting), he/she generates a cost increment to the other users due to interference (marginal cost). The difference between average and marginal costs corresponds to an externality price. Due to negative externality, the optimal point of supply decreases, while the optimal price increases as compared with a scenario without negative externality.

In this case, an increment in the usage of one side (e.g. secondary) will results in additional costs on the other side (e.g. primary). Based on the results depicted in Figure 4 (d) the loss in value for primary users is calculated for an increase in the number of secondary users. These costs correspond to an externality price $\left(\mathrm{P}_{\mathrm{e}}\right)$, which are shown in Figure 5 as an increase (\%) from unit price. This graph reveals that the externality price increases exponentially with the number of users. In fact, $P_{e}$ is relatively low until a certain number of users (approx. 26). From this point, the interference price increases reaching an increase of $90 \%$ from the unit price. Note that these results assume that the same unit price for primary and secondary users and a fixed base station transmission power. The results suggest that the spectrum holder will accept secondary users only if they are willing to pay an extra charge due to the interference caused to the primary users. Otherwise, the spectrum holder will prefer to accept additional primary users, which in this example do not interfere to each other or to increase its transmission power to compensate the caused interference to primaries.

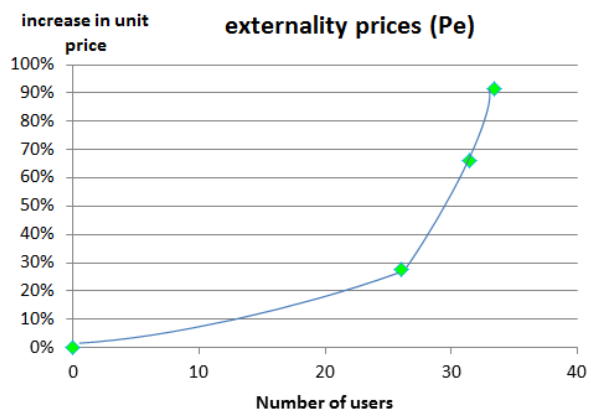

Figure 5: externality prices for secondary access

\section{CONCLUSIONS}

This paper assesses the benefits of flexibility provided by the primary spectrum owner in return for compensation. The simulated scenarios show that the optimal level of interference is usually positive (not zero); therefore, spectrum trading should involve a trade-off decision regarding the amount of interference a spectrum holder is able to receive and generate. In this context, a spectrum transaction should effectively consider the measuring and pricing of the interference.

A flexible spectrum regime should provide the primary operator an economic incentive, which exceeds the value loss due to the interference received by primary users. An optimal 
contract structure should in this case reflect both the primary and secondary user utility functions. In the analyzed scenario, the primary spectrum holder makes the decision on the optimal level of interference, and therefore revenues coming from secondary users should be transferred as additional profits to the primary operator. Additionally, if the spectrum valuation of the secondary is unknown, the price of the secondary access should include an externality charge, which equals the marginal costs due to the externality caused to the primary.

In general, given a spectrum size, an increase in the demand will result in additional gains in a scheme, which allows voluntary transactions with respect to a scheme, which allows no or minimize the interference. In the simulated scenario, an increase in the interference coming from the secondary users, (due to increase in the leasing distance or in the number of users) should result in additional profits for the spectrum holder, even though it generates a drop in the primary SINR.

This study suggests that contract structure should consider to the user utility function. In an extreme case, this function can change from one user to another, depending on the service and the market conditions. In practice, the spectrum holder may be able to estimate the utility function of its own customers only. Congestion pricing applied to the secondary access is in this case a useful mean to reveal the spectrum valuation of the secondary users. This profit analysis indicates that a spectrum retail market, which reveals the secondary valuations, is the most beneficial. Moreover, fee compensation by administrative means has the disadvantage of being far more static for revealing the value of the spectrum. Alternatively, a revenue sharing mechanism such as the already existing between mobile network operators and mobile virtual network operators may be a good first step towards a better utilization of the spectrum.

Although this paper has analyzed a concrete primarysecondary scenario based on a LTE primary system and P2P secondary communication secondary system coexistence in the $700 \mathrm{MHz}$ frequency band, the results are not only specific to this concrete scenario. Indeed, they highlight in general terms the importance of interference management to achieve an optimal social benefit in mobile communications.

\section{ACKNOWLEDGMENT}

This work has also taken place in the context of COST Action IC0905 TERRA, and with partial support of ICTACROPOLIS and TRIAL EECRT projects. The authors wish to thank Wataru Yamada and Ricardo Paredes for their valuable comments and inputs.

\section{REFERENCES}

[1] Klessmann, C.; Nabe, C. and Burges, K. Pros and cons of exposing renewables to electricity market risks - A comparison of the market integration approaches in Germany, Spain, and the UK. Energy Policy 36, 3646-3661, 2008.

[2] Gandhi, S.; Buragohain, C.; Cao, L.; Zheng, H.; Suri, S. Towards realtime dynamic spectrum auctions. Computer Networks 52, 879-897, 2008.
[3] Wang, X.; Li, Z.; Xu, P.; Xu, Y., Gao, X. and Chen, H-H. Spectrum Sharing in Cognitive Radio Networks - An Auction based Approach. IEEE Transactions on Systems, Man and Cybernetics, PART B 1, October 2009.

[4] Niyato, D.; Hossain, E. \& Han, Z. Dynamics of Multiple-Seller and Multiple-Buyer Spectrum Trading in Cognitive Radio Networks: A Game-Theoretic Modeling Approach. IEEE Transactions on Mobile Computing, vol. 8, No. 8., August 2009.

[5] Mastroeni, L.; Naldi, M. Pricing of spectrum reservation under overbooking. Electronic Commerce Research and Applications 10, 565575, 2011.

[6] European Commission, EU COM (2012) 478. European Economic and Social Committee, "Promoting the shared use of radio spectrum resources in the internal market", Brussels, September 2012.

[7] Electronic Communications Committee (ECC). "Light Licensing, License-exempt and Commons", Report 132, June 2009.

[8] Holland, O; De Nardis, L; Nolan K; Medeisis, A; Anker, P; Minervini, L.F.; Velez, F; Matinmikko, M; Sydor, J. Pluralistic Licensing. IEEE International Symposium on Dynamic Spectrum Access Networks (DySPAN), 2012.

[9] Gao, L.; Wang, X.; Xu, Y. and Zhang, Q. Spectrum Trading in Cognitive Radio Networks: A Contract-Theoretic Modeling Approach. IEEE Journal on Selected Areas in Communications, Vol. 29, No. 4, April 2011.

[10] Al Daoud, A.; Murat, A. and Starobinski, D. Pricing strategies for spectrum lease in secondary markets. IEEE/ACM Transactions on Networking (TON), 18 (2), 462-475, 2010.

[11] Al Daoud, A.; Murat, A., and Starobinski, D. Economic viability of private commons: Framework and guidelines for profitability. Telecommunications Policy 37(2), 231-240, 2013.

[12] Umar, R.; Sheikh, A. U.H. A comparative study of spectrum awareness techniques for cognitive radio oriented wireless networks. Physical Communication 9, 148-170, 2013.

[13] Akyildiz, I.F.; Lo, B.F.; Balafrishnan, R. "Cooperative spectrum sensing in cognitive radio networks: A survey". Physical Communication 4, 4062,2011 .

[14] Electronic Communications Committee (ECC). "Technical and operational requirements for the operation of white space devices under geo-location approach", Report 186, January 2013.

[15] Federal Communications Commission, FCC 10-174. Second Memorandum Opinion and Order. Appendix B: Final Rules. Released and Adopted: September 23, 2010.

[16] ETSI TS 103095 V1.1.1. Reconfigurable Radio Systems (RRS); Radio Reconfiguration related Architecture for Mobile Devices, 2013.

[17] Macal, C.M. and North, M.J. Tutorial on agent-based modeling and simulation. Journal of Simulation 4, 151-162, 2010.

[18] Cichon, D. J.; Kürner, T. Final report for COST Action 231, Chapter 4: Propagation Prediction Models. Hata propagation model for urban areas, 1999.

[19] ITU-R P.1411-6. Recommendation. Propagation data and prediction methods for the planning of short-range outdoor radiocommunication systems and radio local area networks in the frequency range $300 \mathrm{MHz}$ to $100 \mathrm{GHz}, 2012$.

[20] Lu, N. Utility-based Bandwidth Adaptation for QoS Provisioning in Multimedia Wireless Networks. Doctoral Thesis. Queen Mary, University of London. Department of Electronic Engineering. UK. April 2007.

[21] 3GPP TR 25.814 V7.1.0. 3rd Generation Partnership Project; Technical Specification Group Radio Access Network; Physical layer aspects for evolved Universal Terrestrial Radio Access (UTRA), Release 7. September 2006

[22] EARTH project. Derivable D2.3: Energy efficiency analysis of the reference systems, areas of improvements and target breakdown. 2010.

[23] Courcoubetis, C; Weber, R. Pricing Communication Networks: Economics, Technology and Modelling. Chapter 9: Congestion. John Wiley \& Sons, Ltd. ISBN: 0-470-85130-9. 2003. 